\title{
Effect of Adamantyl Compounds on Dynamics of Spin Labelled Multilamellar Liposomes
}

\author{
Krunoslav Mirosavljević,, ${ }^{a, b}, *$ Marija Matković, ${ }^{\mathrm{c}}$ Kata Mlinarić-Majerski, ${ }^{\mathrm{c}}$ and Vesna Noethig-Laslo \\ ${ }^{a}$ College of Slavonski Brod, Dr. Mile Budaka 1, HR-35000 Slavonski Brod, Croatia \\ ${ }^{\mathrm{b}}$ Department of Physical Chemistry, Ruđer Bošković Institute, Bijenička cesta 54,10000 Zagreb, Croatia \\ ${ }^{\mathrm{c}}$ Department of Organic Chemistry and Biochemistry, Ruđer Bošković Institute, Bijenička cesta 54, 10000 Zagreb
}

RECEIVED NOVEMBER 22, 2013; REVISED APRIL 11, 2014; ACCEPTED JULY 23, 2014

\begin{abstract}
Interactions of two adamantyl molecules: 3-(adamantan-1-yl)propan-1-aminium chloride (compound A) and 1-hydroxyadamantan (compound B) with multilayer liposomes composed of L- $\alpha$-phosphatidylcholine, cholesterol and dicetylphosphate, spin labelled with $n$-doxylstearic acids $(n=5$, $7,10,12,16)$ were investigated. In the presence of aminium salt $(\mathbf{A})$ or alcohol (B) the increased dynamics of 5-doxylstearic acids and 16-doxylstearic acids in the multilamellar liposomes were determined in the temperature range from $250 \mathrm{~K}$ to $295 \mathrm{~K}$. The increased dynamics of 5-doxylstearic acids suggested that both adamantyl compounds influenced molecular motions in the interfacial region of the liposome. The increased motional properties of 16-doxylstearic acids, located in the core of the multilamellar liposomes, suggested that both adamantyl compounds induced the structural changes in the hydrophobic core as well.
\end{abstract}

Keywords: adamantyl compounds, drug transport, drug interactions, physicochemical properties, spin labelling, ESR, spectroscopy, liposomes, phospholipids

\section{INTRODUCTION}

Multidimensional value of adamantyl group in the drug design has been pointed out in the several recent review papers. ${ }^{1}$ Adamantane is characterised by its rigid and spherical structure ${ }^{2}$, possessing high lipophilicity. ${ }^{3}$ It is well known that introducing an adamantyl moiety, with its unique physicochemical properties, into substances with known biological activity, improves their pharmacological properties and enhances their activity. ${ }^{3,4}$

The synthesis of adamant-2-yl-tripeptides, which belong to the class of immunomodulating compounds comprising the elements of the bacterial peptidoglycan structures, was reported. ${ }^{5}$ These adamantyltripeptides were incorporated into the liposomes. ${ }^{6}$ In order to be close to the cell surface polarity the negatively charged multilamelar liposomes composed of egg-phosphatidylcholine, cholesterol and dicethylphosphate (egg-PC, CHL, DHP), spin labelled with $n$-doxylstearic acids ( $n=$ $5,10,16)$ were used. Interactions of adamantyltripeptides with the liposomes caused increased motional properties of the $n$-doxyl stearic acids in liposomes, even when nitroxide moiety was located at the end of the fatty acid chain (at $16^{\text {th }}$ carbon atom). However, the precise role of the adamantyl moiety in interactions with the liposomes remained unanswered.

Until now, interactions of adamantyl compounds were studied with liposomes composed only of phospholipids. The two possible binding sites of the spin labelled amantadine (at $-\mathrm{NH}_{2}$ group on the amantadine molecule) on the lipid bilayer composed of L- $\alpha$-DMPC or L- $\alpha$-DPPC or L- $\alpha$-DSPC were suggested: in the phospholipid head group region and only a small amount in the hydrophobic centre of the bilayer. ${ }^{7}$ Neutron and $\mathrm{X}$-ray diffraction studies of the amantadine in the complex with DOPC suggested that majority of amantadine molecules interacted with the lipid head groups at the surface of the bilayer and only minor number of molecules penetrated deeper into the bilayer. ${ }^{8}$ Molecular dynamics simulations were used to calculate the potential of mean force of the three different adamantane derivatives (amantadine, rimantadine, memantadine) with the lipid bilayer composed of DOPC. ${ }^{9}$ The results demonstrated preference for the interfacial region of the bilayer for both protonated as well as neutral forms, while permeant species were only the small number of the neutral forms of all the three adamantanes.

\footnotetext{
* Author to whom correspondence should be addressed. (E-mail: krunoslav.mirosavljevic@gmail.com)
} 


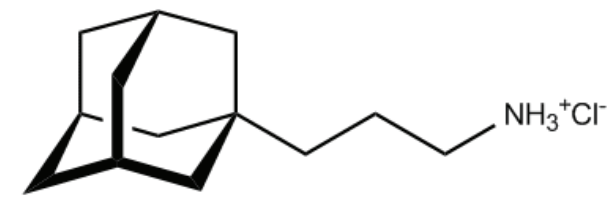

A

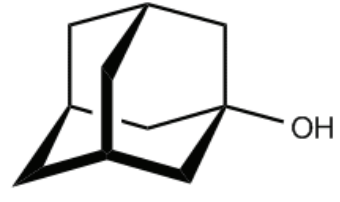

B

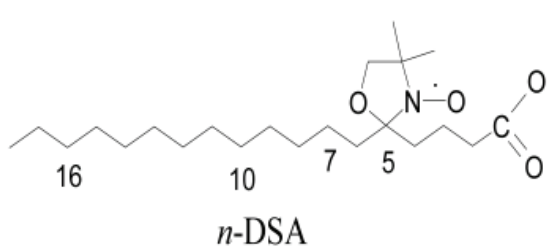

Figure 1. Structural forms of the molecules used in the study: 3-(adamantan-1-yl)propan-1-aminium chloride (A), 1-hydroxyadamantan (B) and $n$-DSA.

Since the discovery of liposomes, liposome-drug delivery systems have played a significant role in the formulation of potent drugs to improve therapeutics. ${ }^{10}$ Although the lipid constituents of liposomes were varied, the presence of cholesterol was essential for the stability of the liposomes.

It was reported in the literature that in the presence of cholesterol and phospholipids with negative charge bind different number of water molecules to the surface of liposomes. ${ }^{11,12}$ Thus, negative charge and saturated fatty acid chains of DHP may influence properties of liposomes and so binding of drugs.

The role of cholesterol on interactions in the liposomes with adamantyl compounds was not studied until now. It is known that presence of cholesterol in the liposomes influenced the polarity profile of liposomes, induced formation of liquid-ordered and liquiddisordered domains and increased the penetration depth of the water molecules into the liposome. ${ }^{10,13,14}$

In order to get more information on the possible role of the adamantyl moiety in interactions with the liposomes that contain cholesterol and negative charge, two different types of adamantyl molecules: 3-(adamantan-1-yl)propan-1-aminium chloride (Figure 1, A) and 1-hydroxyadamantan (Figure 1, B) were studied in this work. Compound $\mathbf{A}$ is the molecule in the form of salt in which the $-\mathrm{NH}_{3}{ }^{+} \mathrm{Cl}^{-}$group and adamantane moiety are separated by three carbon atoms, while compound $\mathbf{B}$ contains only an $-\mathrm{OH}$ group.

\section{EXPERIMENTAL}

\section{Preparation of Spin Labelled Liposomes}

L- $\alpha$-Phosphatidylcholine, type XI-E: from fresh egg yolk (egg-PC), cholesterol from porcine liver (CHL), dicetylphosphate (DHP), the spin labelled fatty acids: $n$-doxylstearic acids ( $n$-DSA, Figure 1$)(n=5,7,10,12$, 16), were purchased from Sigma Chemical Co. (St. Louis, MO, USA).

Egg-PC $28.4 \mathrm{mg}(0.35 \mathrm{mmol}) ; \mathrm{CHL} 9.8 \mathrm{mg}$ $(0.25 \mathrm{mmol})$ and DHP $2.7 \mathrm{mg}(0.05 \mathrm{mmol})$, giving a molar ratio of $7: 5: 1$ with 1 molar $\%$ of $n$-doxylstearic acids ( $n$-DSA, $n=5,7,10,12,16)$ were dissolved in
$4 \mathrm{~mL}$ of $\mathrm{CHCl}_{3}$. After rotary evaporation of the solvent, the remaining lipid film was dried in vacuum for an hour and then dispersed by vigorous hand shaking in $2.5 \mathrm{~mL}$ phosphate buffered saline (PBS) at $\mathrm{pH} 7.4$ with or without $5 \mathrm{mM}$ adamantyl compounds (compound $\mathbf{A}$ or B). Results with two different liposome preparations are presented.

\section{Synthesis}

3-(adamantan-1-yl)propan-1-aminium chloride (compound A) was prepared from 3-(adamantan-1-yl)propionitrile by method of direct hydrogenation as follows: to the solution of 3-(adamantan-1-yl)-propionitrile $(337 \mathrm{mg}, 1.78 \mathrm{mmol})$ in dry EtOH $(40 \mathrm{~mL}), \mathrm{CHCl}_{3}$ $(1.90 \mathrm{~mL})$, conc. $\mathrm{HCl}(0.90 \mathrm{~mL})$ and $\mathrm{PtO}_{2}(50 \mathrm{mg})$ were added. ${ }^{15}$ The reaction mixture was subjected to hydrogen pressure (63-50 psi, Parr apparatus) at room temperature for 2 days. Analysis by thin layer chromatography $\left(\mathrm{CH}_{2} \mathrm{Cl}_{2}\right.$, with iodine chamber used for visualization of the starting compound) indicated complete consumption of the starting nitrile. The completion of the reaction was also confirmed by using gas chromatography. The reaction mixture was filtrated (,Whatman“ filter paper; $11.0 \mathrm{~cm}, 44$ ), the solvent evaporated almost to the dryness and diethyl ether was added $(40 \mathrm{~mL})$ which produced white precipitate. The precipitate was filtered off on sintered glass filter and was washed with diethyl ether. The filtrate was evaporated almost to the dryness and $\mathrm{CH}_{2} \mathrm{Cl}_{2}(5 \mathrm{~mL})$ followed by diethyl ether $(30 \mathrm{~mL})$ added, to produce another amount of white precipitate. The collected precipitate portions afforded final aminium chloride A $(254 \mathrm{mg})$ in $62 \%$ yield. The structure and the purity of the prepared compound were confirmed by NMR spectroscopy (Supplement, Figures S1 and S2). The pKa value of compound $\mathbf{A}$ has been calculated (Supplement, p. 4) and also experimentally determined by pH titration (Supplement, p. 5-6) as 10.20 and 9.76 respectively. The difference between the theoretical and experimental value of $\mathrm{pKa}(\mathbf{A})$ is probably due to low solubility of conjugated base of $\mathbf{A}$ (amine) which causes visible precipitation that drives forward the process of deprotonation, lowering the $\mathrm{pKa}(\mathbf{A})$ value. However, both of the obtained values are in the usual pKa range for aminium compounds. 
1-hydroxyadamantan (compound B) was prepared from 1-bromoadamantane by the standard literature procedure as follows: to the solution of 1-bromoadamantane $(300 \mathrm{mg}, 1.39 \mathrm{mmol})$ in DMF $(1.30 \mathrm{~mL})$, $0.67 \mathrm{M} \mathrm{HCl}(0.45 \mathrm{~mL})$ was added. ${ }^{16}$ The obtained suspension was diluted with water $(1 \mathrm{~mL})$ and heated to $105{ }^{\circ} \mathrm{C}$. The heating was continued for additional 30 minutes at $102-110{ }^{\circ} \mathrm{C}$. To the cooled reaction mixture water $(10 \mathrm{~mL})$ was added, formed precipitate filtrated off on sinter glass and was washed with water $(5 \times 5 \mathrm{~mL})$. The obtained white precipitate was dissolved in $\mathrm{CH}_{2} \mathrm{Cl}_{2}(40 \mathrm{~mL})$ and washed with water $(15 \mathrm{~mL})$. The organic phase was dried over anhydrous $\mathrm{MgSO}_{4}$ for 15 minutes, filtrated and the solvent removed under reduced pressure to obtain final product $\mathbf{B}$ as white solid (146 mg) in $69 \%$ yield. The structure and the purity of the prepared compound were confirmed by NMR spectroscopy (Supplement, Figures S3 and S4), GC analysis (capillary column $\mathrm{DB} 210^{\circ}$ ) and analytical thin layer chromatography on silica gel $\left(7 \% \mathrm{MeOH}\right.$ in $\mathrm{CH}_{2} \mathrm{Cl}_{2}$, $\mathrm{R}_{f}=0.42$, visualisation in iodine).

\section{ESR Measurements}

ESR measurements were done on Varian E-9 spectrometer $(10 \mathrm{GHz})$ equipped with a Bruker variable temperature control unit. The spectra were recorded with digital acquizition, EW-ESR ware. ${ }^{17}$ Sample capillaries were inserted into the standard $4 \mathrm{~mm}$ diameter ESR quartz tubes and centred in a TE102 ESR cavity. At each temperature, the samples were left for about 10 minutes for temperature equilibration.

ESR spectra of $n$-doxylstearic acids ( $n$-DSA) in liposomes reflect motional properties of the lipid bilayer. One important motional property is the librational motion of the $>\mathrm{N}-\mathrm{O}$ moiety of the nitroxide bound on the fatty acid about a direction near perpendicular to the bilayer surface. This motional effect is qualitatively measured by the ESR experimental parameters: maximal, $A_{\max }(\mathrm{mT})$, and minimal, $A_{\min }(\mathrm{mT})$, of the hyperfine splitting in the spectra as is denoted in Figures 2 and 4. The differences between ESR spectra of multilamellar liposomes that originate from two preparations are due to the fact that preparation of spin labelled liposomes is very sensitive process and one can rarely expect to prepare two completely identical liposomes. However, presented work emphasizes the relative differences in ESR spectra due to the presence of adamantyl compounds A or B compared to multilamellar liposomes without them.

\section{RESULTS AND DISCUSSION}

ESR spectra of $n$-DSA ( $n=5,7,12$ and 16) in the multilamellar liposomes with and without incorporated 3-(adamantan-1-yl)propan-1-aminium chloride (compound A) recorded at $273 \mathrm{~K}$ are shown in Figure 2. $2 A_{\max }$ and $2 A_{\min }$ parameters are denoted with arrows. $2 A_{\max }$ determined for the spectra of 5-DSA in the multilamellar liposomes with compound A was $6.05 \mathrm{mT}$, while without $6.29 \mathrm{mT}$ (spectrum A). The observed difference is due to faster librational motion of the nitroxide moiety in the presence of 3-(adamantan-1yl)propan-1-aminium salt (compound A) and suggests the effect of the adamantyl group at the $5^{\text {th }}$ position along the fatty acid chain in the liposomes i.e., near the liposome's negatively charged surface. Calculated and experimental value of $\mathrm{pKa}(\mathbf{A})$ determined as 10.20 and 9.76 respectively, revealed that compound $\mathbf{A}$, in given experimental conditions (PBS, pH 7.4), is mostly in protonated form which would prefer location of adamantyl part of molecule in upper part of liposomal membrane.

ESR spectra of 7-DSA as well as of 12-DSA remained unchanged in the presence of the compound $\mathbf{A}$ (Figure 2, spectra B and C). On the other hand, even at $273 \mathrm{~K}$, in the presence of aminium salt (compound A), 16-DSA showed increased librational motion of the spin label, although this spin label is located in the hydrophobic core of the liposomes. $2 A_{\max }$ of 16-DSA without incorporated adamantyl molecule is $3.95 \mathrm{mT}$ and with incorporated adamantyl molecule is $3.57 \mathrm{mT}$ (Figure 2, spectrum D). This result suggests an influence of the compound $\mathbf{A}$ on the hydrophobic core of the liposome.

ESR spectra of $n$-DSA $(n=5,7,12$ and 16) in the multilamellar liposomes with and without incorporated compound $\mathbf{A}$ were recorded in the temperature range from $183 \mathrm{~K}$ to $298 \mathrm{~K}$. $2 A_{\max }$ parameter as a function of the temperature is presented in Figure 3 . The difference in $2 A_{\max }$ values for the spectra of 5-DSA with (empty

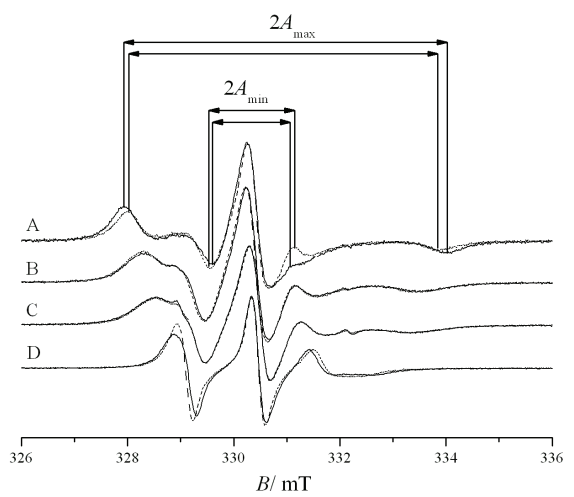

Figure 2. ESR spectra of the spin labels (A - 5-DSA; $\mathrm{B}$ - 7-DSA; C - 12-DSA; D - 16-DSA) in the multilamellar liposomes (composed of egg-PC/CHL/DHP) with (dotted line) and without (full line) 3-(adamantan-1-yl)propan-1-aminium chloride (compound A). $2 A_{\max }$ and $2 A_{\min }$ parameters are denoted. All spectra were taken at $273 \mathrm{~K}$. 
squares) and without (full squares) the addition of 3-(adamantan-1-yl)propan-1-aminium chloride were determined above $250 \mathrm{~K}$, i.e. above the temperature at that water molecules bound at the liposome's surface started to be liquid. There were no differences in $2 A_{\max }$ parameters for the spectra of 7-DSA (triangles) or 12-DSA (circles) between the liposomes with and without incorporated compound $\mathbf{A}$ in whole temperature range measured.

For the spectra of 16-DSA (rhombs), the difference in the temperature dependence of $2 A_{\max }$ parameters was observed at similar temperatures as for 5-DSA. This fact suggests that location of the compound $\mathbf{A}$ near the $5^{\text {th }}$ carbon atom of the fatty acid chain also influenced physical properties at the hydrophobic core of the liposome. One possibility is that appearance of the adamantane in the interfacial region of the negatively charged liposome induced changes in the lipid ordered/ liquid disordered domain (influenced also by cholesterol presence), as well as some conformation change in the hydrophobic core of the liposomes.

Compound B (1-hydroxyadamantan) is a neutral molecule with the $-\mathrm{OH}$ group directly attached to the adamantane moiety. ESR spectra of four different spin labels, $n$-DSA ( $n=5,7,10$ and 16) in the multilamellar liposomes (egg-PC/CHL/DHP) with (dotted line) and without (full line) incorporated 1-hydroxyadamantan, recorded at $273 \mathrm{~K}$, are shown in Figure 4. At $273 \mathrm{~K}$, $2 A_{\max }$ of 5-DSA without incorporated 1-hydroxyadamantan is $6.08 \mathrm{mT}$ while with incorporated $\mathbf{B}$ is $5.91 \mathrm{mT}$. The observed difference is due to faster librational motion of the nitroxide moiety at the $5^{\text {th }}$ position along the fatty acid chain in the presence of the compound B. It is interesting that the same result was obtained as in the case of compound $\mathbf{A}$.

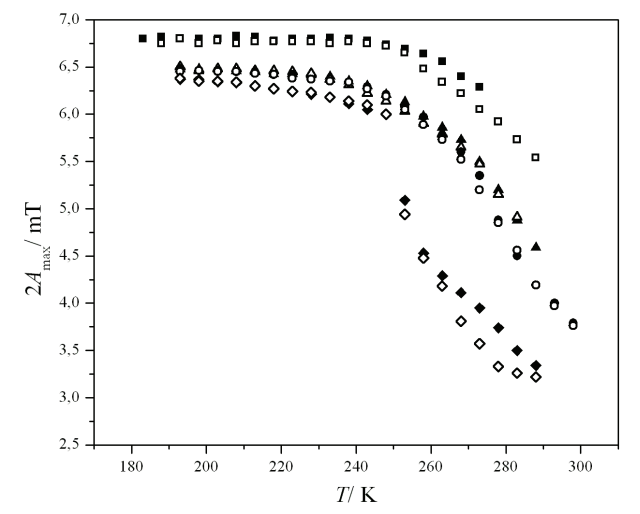

Figure 3. Temperature dependence of $2 A_{\max }$ parameter of the spin labels: 5-DSA - squares, 7-DSA - triangles, 12-DSA circles, 16-DSA - rhombs in the multilamellar liposomes (egg-PC/CHL/DHP) with (empty signs) and without (full signs) the addition of 3-(adamantan-1-yl)propan-1-aminium chloride (compound $\mathbf{A}$ ).
ESR spectra of 7-DSA in the multilamellar liposomes with compound B doesn't show the difference from ESR spectra of 7-DSA in the multilamellar liposomes without compound B (Figure 4, spectra B) but they differ from those with compound $\mathbf{A}$ due to the different functional groups of compounds A (charged, with hydrocarbon tail) and $\mathbf{B}$ (neutral, capable $\mathrm{OH}$ hydrogen bonding). This difference is also seen at several low temperatures (Figures 3, 5A and 5B). Differences in $2 A_{\max }$ and $2 A_{\min }$ parameters for 16-DSA, shown in spectrum $\mathrm{D}$, are also similar as in the case of compound $\mathbf{A}$. The $2 A_{\max }$ parameter of 16 -DSA without incorporated 1-hydroxyadamantan is $3.81 \mathrm{mT}$ (full rhombs) while with incorporated adamantyl molecule is $3.54 \mathrm{mT}$ (empty rhombs) at $273 \mathrm{~K}$. This result suggests the influence of the 1-hydroxyadamantan on the physical properties of the hydrophobic core.

ESR spectra of four different $n$-DSA $(n=5,7,10$ and 16) in the multilamellar liposomes with and without incorporated 1-hydroxyadamantane (compound B) were recorded in the temperature range from $183 \mathrm{~K}$ to $293 \mathrm{~K}$, i.e. in the same temperature range as in the experiments with adamantane aminium salt (compound $\mathbf{A}$ ). The temperature dependence of $2 A_{\max }$ parameters are shown in Figures $5 \mathrm{~A}$ and $5 \mathrm{~B}$. The difference in the values of $2 A_{\max }$ for the spectra of 5-DSA with (empty squares) and without (full squares) 1-hydroxyadamantan (compound B) began at around $255 \mathrm{~K}$ and were recorded at all higher temperatures (Figure 5A). For the spectra of 16-DSA (Figure 5B, rhombs), the differences in the temperature dependence of $2 A_{\max }$ parameter could be determined above $250 \mathrm{~K}$ in the area where the bulk water in liposomes is starting to melt down. These findings are similar to those obtained for compound $\mathbf{A}$ (shown in Figure 3).

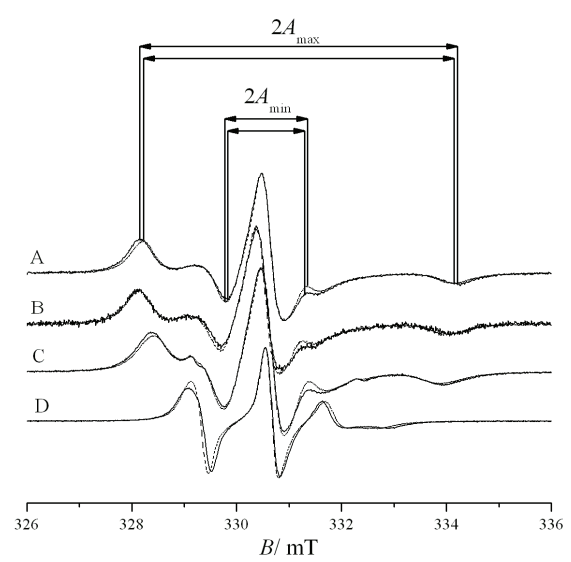

Figure 4. ESR spectra of the spin labels (A - 5-DSA; B - 7-DSA; C - 10-DSA; D - 16-DSA) in the multilamellar liposomes with (dotted line) and without (full line) the addition of 1-hydroxyadamantan (compound B). $2 A_{\max }$ and $2 A_{\min }$ parameters are denoted. All spectra were taken at $273 \mathrm{~K}$. 
(a)

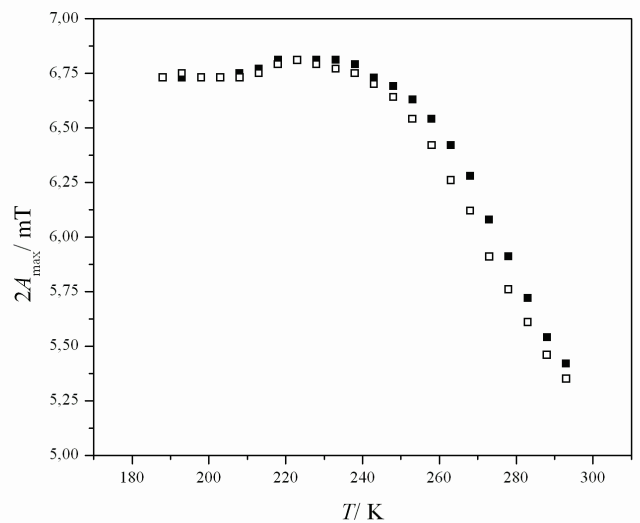

(b)

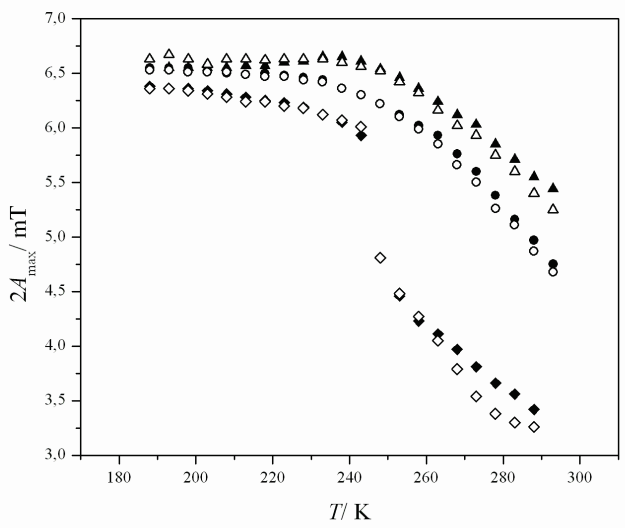

Figure 5. (a) Temperature dependence of $2 A_{\max }$ parameter of 5-DSA in multilamellar liposomes with (empty signs) and without (full signs) 1-hydroxyadamantan (compound B); (b) Temperature dependence of $2 A_{\max }$ parameter of different spin labels (7-DSA - triangles, 10-DSA - circles, 16-DSA rhombs) in multilamellar liposomes with (empty signs) and without (full signs) incorporated 1-hydroxyadamantan (compound $\mathbf{B}$ ).

It is of interest to note that the two physically and structurally different adamantyl compounds studied in this work gave same results in interaction with liposomes, and similar to the one obtained previously with adamantyltripeptides. ${ }^{4}$ This indicates that location of the studied adamantyl compounds in liposomes is mainly determined by the bulky adamantane moiety.

\section{CONCLUSION}

In the presence of adamantane compounds; 3-(adamantan-1-yl)propan-1-aminium chloride (A) or 1-hydroxyadamantan (B) (Figure 1), increased motional properties of the spin labels: 5-doxylstearic acids located in the interfacial region of the liposome and 16-doxylstearic acids located in the hydrophobic core of the liposome were determined. This result suggests location of the both $\mathbf{A}$ and $\mathbf{B}$ near the $5^{\text {th }}$ carbon atom of the fatty acid chain and influence on the physical properties at the hydrophobic core of the liposomes. One possibility is that appearance of adamantane in the interfacial region of the liposome induced changes in the lipid ordered/liquid disordered domain of the liposomes, as well as that it induced conformation changes at the hydrophobic core of the liposomes.

Acknowledgements. The work was supported by Croatian Ministry of Science, Education and Sport (Project No: 0980982915-2939 and Project No: 098-0982933-2911).

\section{REFERENCES}

1. J. Liu, D. Obando, V. Liao, T. Lifa, and R. Codd, Eur. J. Med. Chem. 46 (2011) 1949-1963.

2. R. C. Fort and P. V. R. Schleyer, Chem Rev 64 (1964) 277-300.

3. J. Joubert, W. J. Geldenhuys, C. J. Van der Schyf, D. W. Oliver, H. G. Kruger, T. Govender, and S. F. Malan, ChemMedChem 7 (2012) 375-384.

4. G. Lamoureux and G. Artavia, Curr. Med. Chem. 17 (2010) 2967-2978.

5. B. Vranešić, J. Tomašić, S. Smerdel, D. Kantoci, and F. Benedetti, Helv. Chim. Acta 76 (1993) 1752-1758.

6. R. Frkanec, V. Noethig-Laslo, B. Vranešić, K. Mirosavljević, and J. Tomašić, Biochim. Biophys. Acta 1611 (2003) 187-196.

7. W. K. Subczynski, J. Wojas, V. Pezeshk, and A. Pezeshk, J. Pharm. Sci. 87 (1998) 1249-1254.

8. J. Wang, J. R. Schnell, and J. J. Chou, Biochem. Biophys. Res. Commun. 324 (2004) 212-217.

9. C. F. Chew, A. Guy, and P. C. Biggin, Biophys. J. 95 (2008) 5627-5636.

10. K. Mirosavljević and V. Noethig-Laslo, Chem. Phys. Lipids $\mathbf{1 5 5}$ (2008) 74-79.

11. D. Bach and I. R. Miller, Biochim. Biophys. Acta 1368 (1998) 216-224.

12. D. Bach and I. R. Miller, Chem. Phys. Lipids 136 (2005) 67-72.

13. V. Noethig-Laslo and M. Šentjurc, Transmembrane Polarity Profile of Lipid Membranes, in: A. Leitmannova Liu, (Ed). Advances in Planar Lipid Bilayers and Liposomes, Volume 5, Elsevier, 2007 pp. 365-415.

14. K. Mirosavljević and V. Noethig-Laslo, Croat. Chem. Acta 81 (2008) 631-636.

15. J. K. Chakrabarti, M. J. Foulis, T. M. Hotten, S. S. Szinai, and A. Todd, J. Med. Chem. 17 (1974) 602-609.

16. H. W. Geluk, and J. L. M. A. Schlatmann, Tetrahedron 24 (1968) 5361-5368.

17. P. D. Morse, Biophys. J. 51 (1987) 440a-495a. 
Supporting material for

Effect of adamantyl compounds on dynamics of spin labelled multilamellar liposomes

\section{Krunoslav Mirosavljevića,b*, Marija Matkovićc ${ }^{c}$ Kata Mlinarić-Majerski ${ }^{c}$, Vesna Noethig-Laslo ${ }^{b}$}

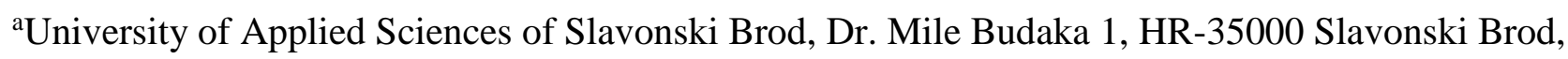
Croatia, e-mail: krunoslav.mirosavljevic@gmail.com

${ }^{\text {b} D e p a r t m e n t ~ o f ~ P h y s i c a l ~ C h e m i s t r y, ~ R u đ e r ~ B o s ̌ k o v i c ́ ~ I n s t i t u t e, ~ B i j e n i c ̌ k a ~ c e s t a ~ 54, ~ P . O . ~ B o x ~ 180, ~}$ HR-10002 Zagreb, Croatia, e-mail: 1aslo@irb.hr

${ }^{\mathrm{c}}$ Department of Organic Chemistry and Biochemistry, Ruđer Bošković Institute, Bijenička cesta 54, P.O. Box 180, HR-10002 Zagreb, Croatia, e-mail: marija.matkovic@irb.hr, kata.majerski@irb.hr *Corresponding author: Krunoslav Mirosavljević, University of Applied Sciences of Slavonski Brod, Dr. Mile Budaka 1, HR-35000 Slavonski Brod, Croatia, phone: 00385-35-492630, fax: 0038535-492804, e-mail: krunoslav.mirosavljevic@gmail.com 
$-\mathrm{NH}_{3}{ }^{+} \mathrm{Cl}^{-}$

A

3-(adamantan-1-yl)propan-l-aminium chloride (A): ${ }^{1} \mathrm{H}$ NMR (DMSO- $\mathrm{d}_{6}$, $300 \mathrm{MHz}): \delta=1.01-1.09(\mathrm{~m}, 2 \mathrm{H}), 1.41-1.47(\mathrm{~m}, 6 \mathrm{H}), 1.48-1.70(\mathrm{~m}, 8 \mathrm{H}), 1.93$ (br s, 3H), 2.69 (t, 2H, $J=7.5 \mathrm{~Hz}$ ), 8.05 (br s, 3H, $\mathrm{NH}_{3}{ }^{+}$) ppm. ${ }^{13} \mathrm{C}$ NMR (DMSO-d $\left.6,75 \mathrm{MHz}\right): 20.4$ $(\mathrm{t}), 27.9(\mathrm{~d}), 31.6(\mathrm{q}), 36.6(\mathrm{t}), 39.6(\mathrm{t}), 40.5(\mathrm{t}), 41.8(\mathrm{t}) \mathrm{ppm}$.

SpinWorks 2.3:

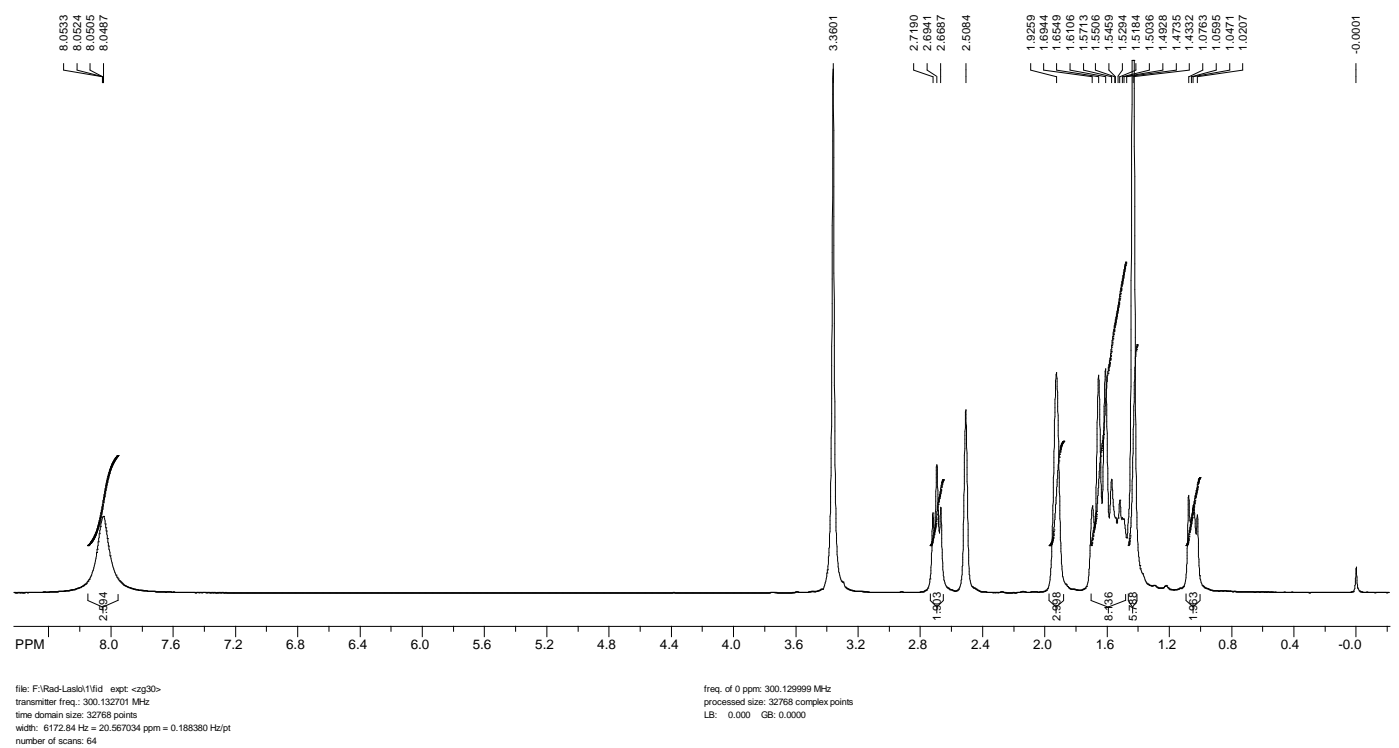

Figure S1: ${ }^{1} \mathrm{H}$ NMR spectrum (DMSO-d $6,300 \mathrm{MHz}$ ) of 3-(adamantan-1-yl)propan-1-aminium chloride (A).

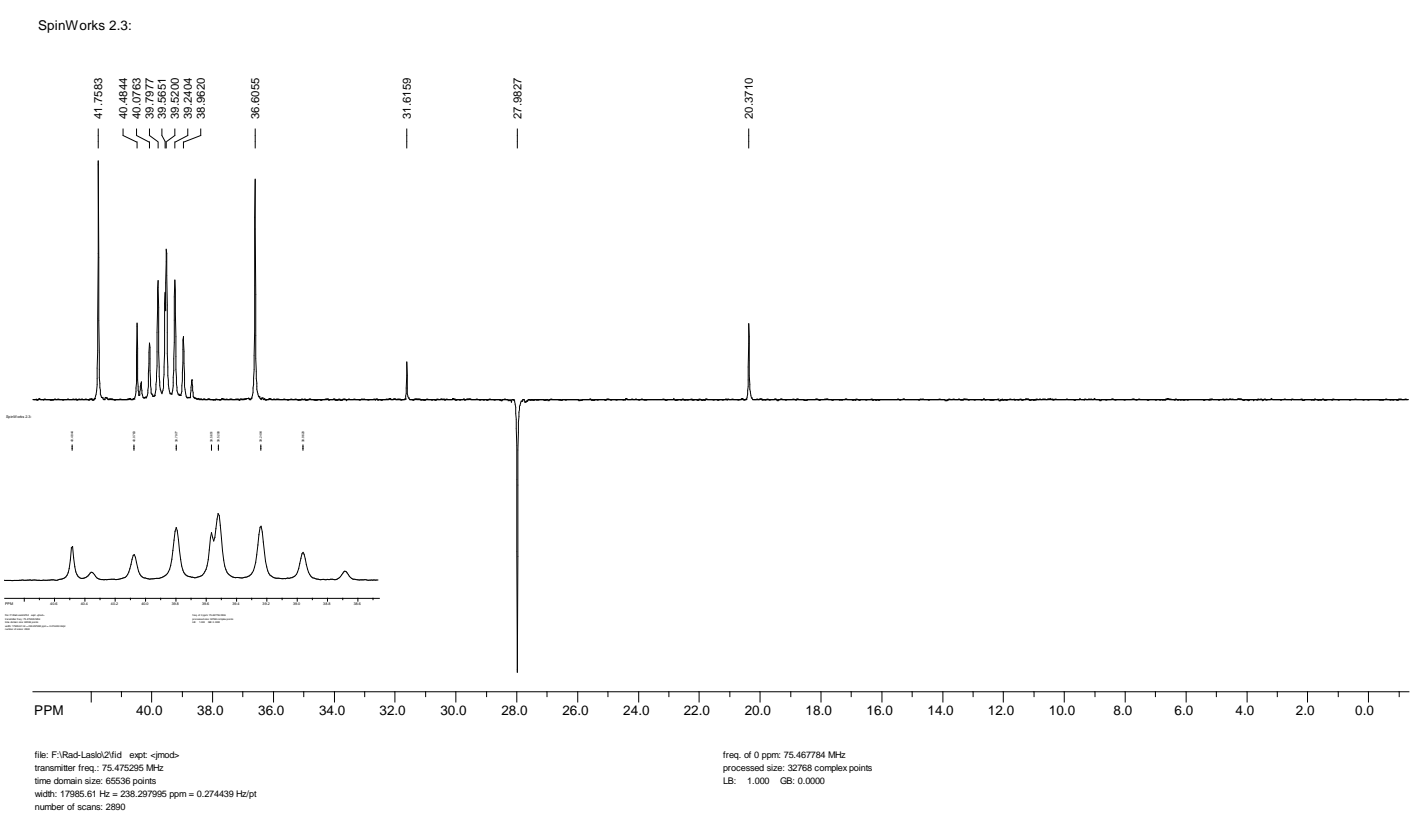

Figure S2: ${ }^{13} \mathrm{C}$ NMR (APT) spectrum (DMSO-d $6,75 \mathrm{MHz}$ ) of 3-(adamantan-1-yl)propan-1aminium chloride (A) 
$-T_{\mathrm{OH}}$

B

1-hydroxyadamantane (B): ${ }^{1} \mathrm{H}$ NMR $\left(\mathrm{CDCl}_{3}, 600 \mathrm{MHz}\right): 1.39(\mathrm{~s}, \mathrm{OH}), 1.57-1.66(\mathrm{~m}$,

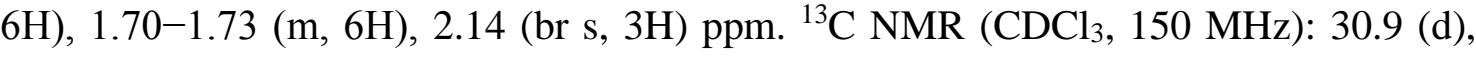
$36.3(\mathrm{t}), 45.6(\mathrm{t}), 68.4(\mathrm{q}) \mathrm{ppm}$.

SpinWorks 2.3:
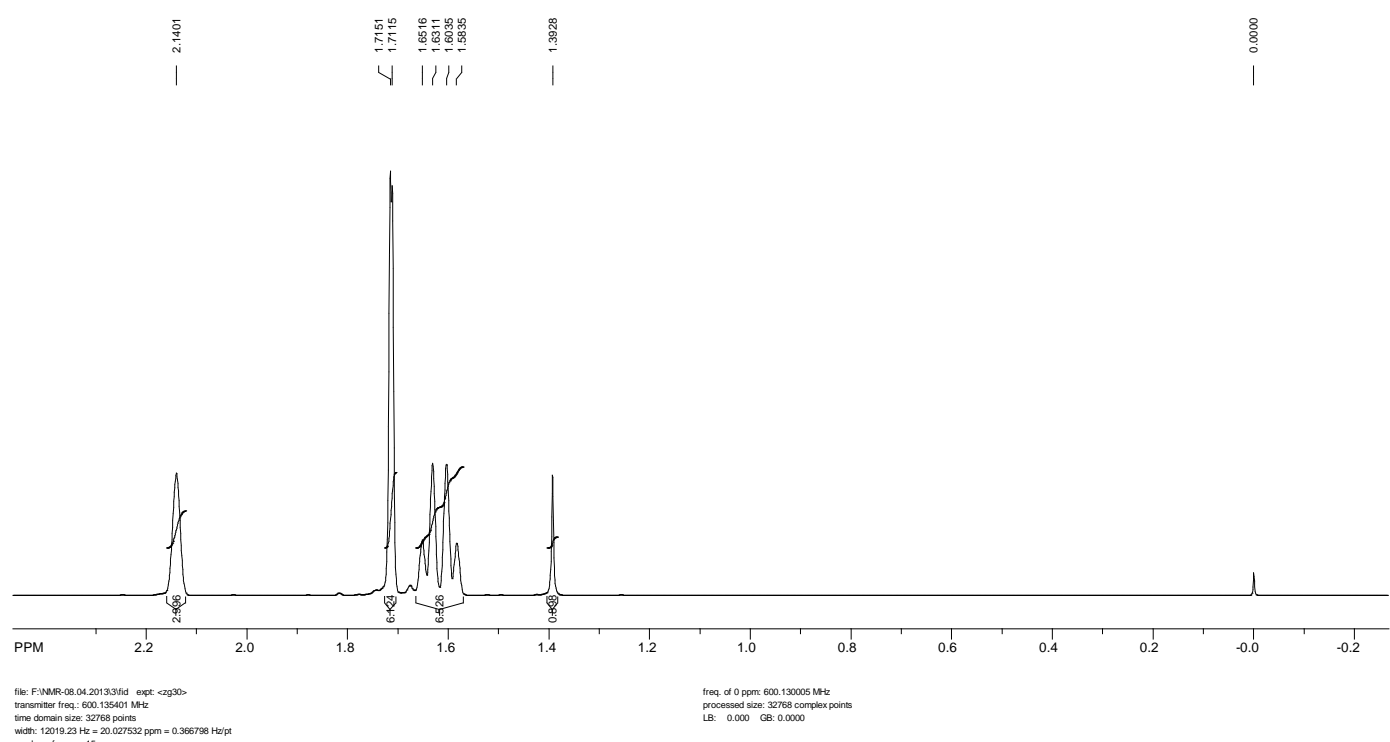

Figure S3: ${ }^{1} \mathrm{H}$ NMR spectrum $\left(\mathrm{CDCl}_{3}, 600 \mathrm{MHz}\right)$ of 1-hydroxyadamantane $(\mathbf{B})$
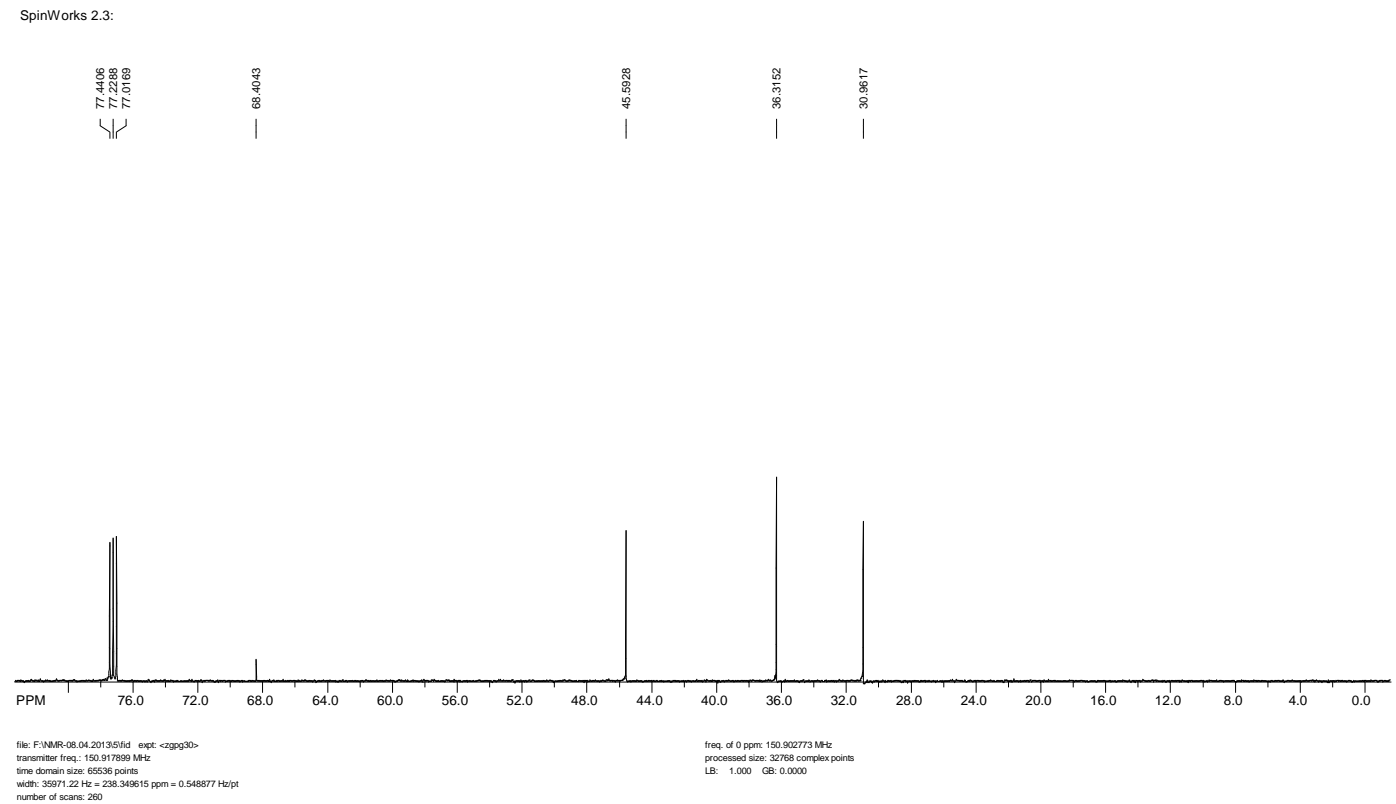

Figure S4: ${ }^{13} \mathrm{C}$ NMR $(\mathrm{COM})$ spectrum $\left(\mathrm{CDCl}_{3}, 150 \mathrm{MHz}\right)$ of 1-hydroxyadamantane $(\mathbf{B})$ 
The calculations where made using ChemAxon's ${ }^{1}$ free softwer MarvinSketch on-line. Calculated pKa of aminium salt $\mathbf{A}$ is 10.20 (Figure S5 compound 2.)

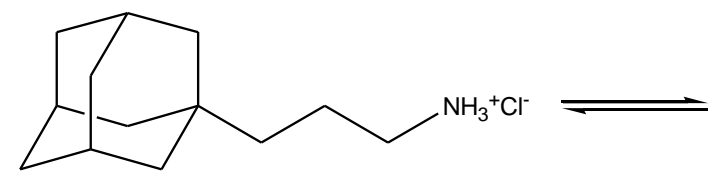

2

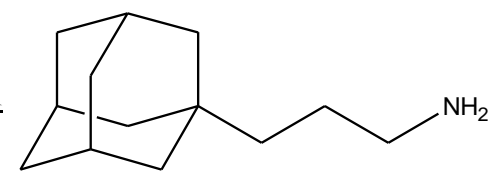

1
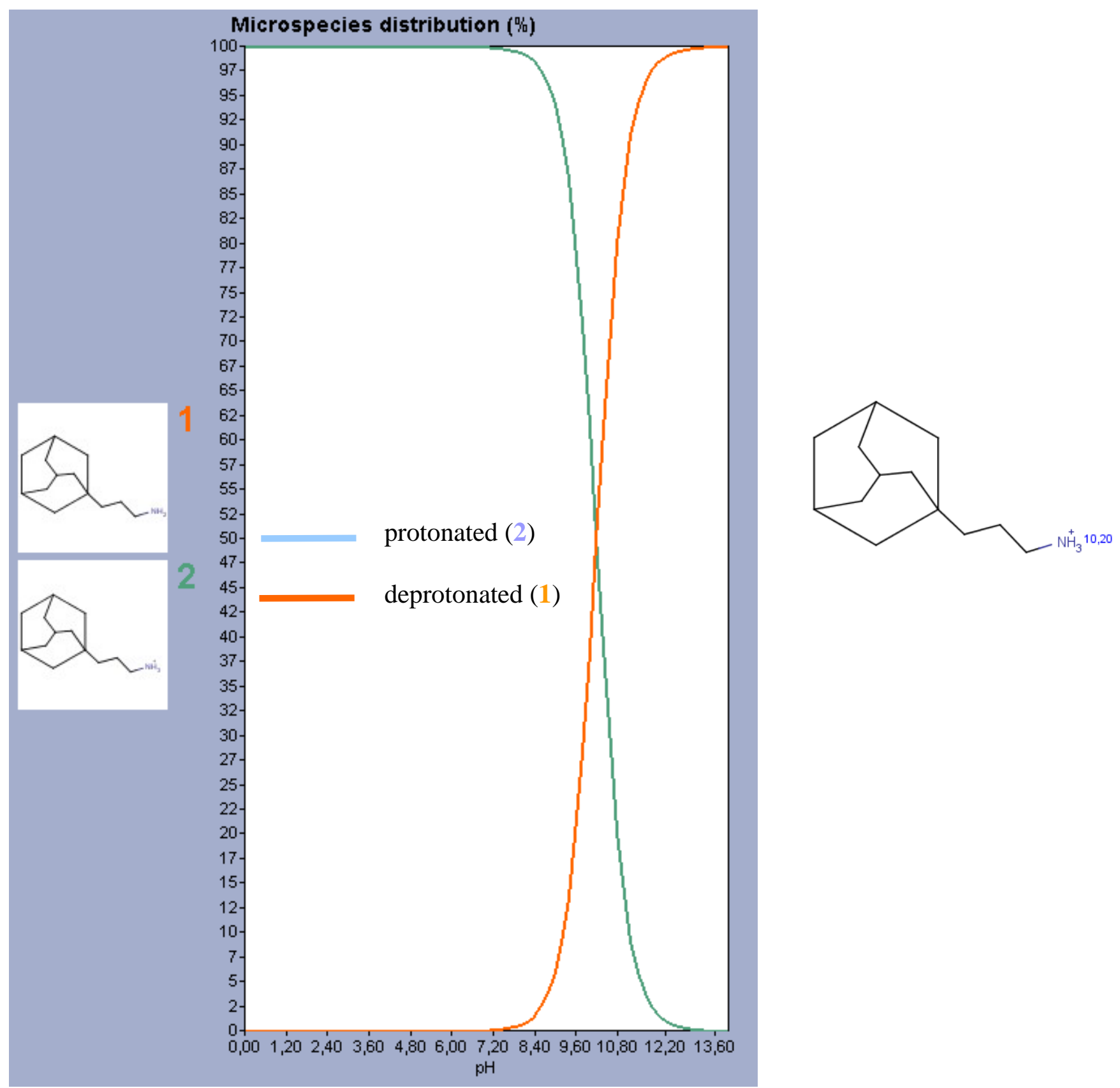

Figure S5: Percentage distrubution of aminium salt $\mathbf{A}$ (2) and its conjugated base (1) in the dependence of $\mathrm{pH}$ value.

${ }^{1}$ http://www.chemaxon.com

02.03.2014, 20.38 
Experimental conditions:

Solution of analyte $(15.9 \mathrm{mM})$ was prepared by dissolving $\mathbf{A}(23.63 \mathrm{mg}, 0.103 \mathrm{mmol})$ in redistilled water $(6.444 \mathrm{~mL})$. The solution was titrated with $0.2 \mathrm{M} \mathrm{NaOH}$ (Table S1). The experimental $\mathrm{pH}$ titration curve (Figure S6 -) fits math. modelled sigmoidal curve (Figure S6 -) with high accuracy $\left(\mathrm{R}^{\wedge} 2=0.99911\right)$ wherein the equivalence point (sigmoidal $\mathrm{X} 0$ point) has been determined at 0.644

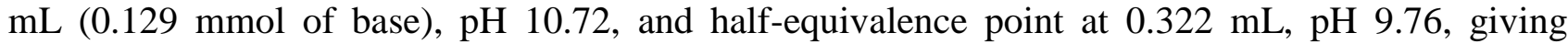
$\operatorname{pKa}(\mathbf{A})=9.76$.

Table S1: pH titration of compound A

\begin{tabular}{|c|c|c|}
\hline Entry & $\mathbf{V}(\mathbf{0 . 2} \mathbf{M ~ N a O H}) / \mathbf{m L}$ & $\mathbf{p H}$ \\
\hline 1 & 0.02 & 8.93 \\
\hline 2 & 0.03 & 9.16 \\
\hline 3 & 0.05 & 9.33 \\
\hline 4 & 0.08 & 9.34 \\
\hline 5 & 0.11 & 9.39 \\
\hline 6 & 0.21 & 9.54 \\
\hline 7 & 0.31 & 9.71 \\
\hline 8 & 0.41 & 9.94 \\
\hline 9 & 0.51 & 10.21 \\
\hline 10 & 0.61 & 10.56 \\
\hline 11 & 0.71 & 10.99 \\
\hline 12 & 0.91 & 11.56 \\
\hline
\end{tabular}




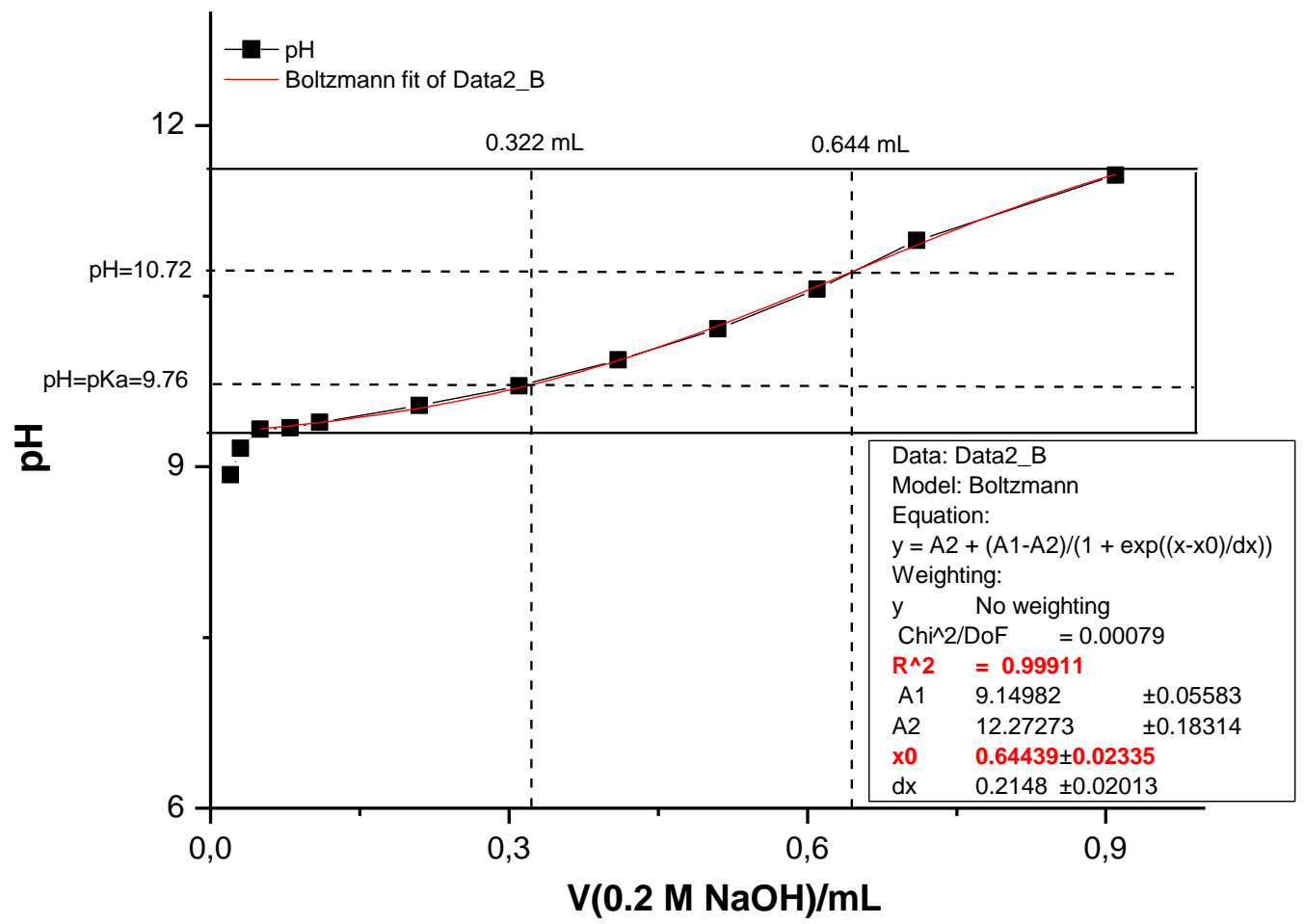

Figure S6: $-\mathrm{pH}$ titration curve of $\mathbf{A}$, with water solution of $\mathbf{A}$ as titrand and $0.2 \mathrm{M} \mathrm{NaOH}$ as titrant.

- sigmoidal curve obtained by Boltzmann fit of experimental values according to sigmoidal curve equitation $y=A 2+(A 1-A 2) /(1+\exp ((x-x 0) / d x))$ 